\title{
Overnight Safety Evaluation of a Multi-Pressure Dial in Eyes with Glaucoma: Prospective, Open-Label, Randomized Study
}

This article was published in the following Dove Press journal: Clinical Ophthalmology

\author{
Tanner J Ferguson (1) \\ Nathan M Radcliffe $\mathbb{D}^{2}$ \\ Sarah H Van Tassel ${ }^{3}$ \\ Brandon J Baartman ${ }^{4}$ \\ Vance M Thompson ${ }^{4}$ \\ Richard L Lindstrom ${ }^{4,5}$ \\ Mitch J Ibach ${ }^{4,5}$ \\ John P Berdahl (D) \\ 'Cole Eye Institute, Cleveland Clinic, \\ Cleveland, OH, USA; ${ }^{2} \mathrm{New}$ York Eye and \\ Ear Infirmary of Mount Sinai, New York, \\ NY, USA; ${ }^{3}$ Department of \\ Ophthalmology, Weill Cornell Medicine, \\ New York, NY, USA; ${ }^{4}$ Vance Thompson \\ Vision, Sioux Falls, SD, USA; ${ }^{5}$ Minnesota \\ Eye Consultants, Minnesota, MN, USA
}

Correspondence: Tanner J Ferguson Email tannerferg@gmail.com
Purpose: To investigate the safety and tolerability of the multi-pressure dial (MPD) worn overnight for seven consecutive days.

Design: Prospective, open-label, randomized, single-site study.

Subjects: Twenty eyes of 10 subjects with open-angle glaucoma were fitted with an MPD and randomized to negative pressure application of $-10 \mathrm{mmHg}$ in one eye (study eye) worn overnight for 7 consecutive days.

Methods: Safety measures included best-corrected visual acuity (BCVA), intraocular pressure (IOP) changes from baseline during and after negative pressure application, slit lamp and dilated fundus exam findings, and rate of adverse events. Subjective assessments were completed daily by the subjects during the 7-day study period and immediately following the study period.

Results: Prior to the 7-day testing period, application of $10 \mathrm{mmHg}$ negative pressure reduced mean IOP from $18.2 \pm 3.8 \mathrm{mmHg}$ to $14.0 \pm 2.1 \mathrm{mmHg}(\mathrm{p}<0.01)$, a $22 \%$ reduction. After 7 days of consecutive nightly wear, repeat IOP measurements with the application of negative pressure showed a decrease in mean IOP from $16.9 \pm 4.3 \mathrm{mmHg}$ to $13.5 \pm$ $3.7 \mathrm{mmHg}$. The observed IOP reduction was in addition to the subjects' current treatment regimen. There were no statistically significant changes in IOP, BCVA from baseline following the 7-day period of nightly wear with the application of negative pressure. The patient-reported outcomes were favorable.

Conclusion: The MPD can safely and comfortably be worn overnight. The decrease in IOP of $>20 \%$ in addition to current therapy is both clinically and statistically significant. The MPD shows promise as a potential new treatment option for nocturnal IOP control.

Keywords: multi-pressure dial, open-angle glaucoma, normal-tension glaucoma, nocturnal IOP, multi-pressure glaucoma management

\section{Introduction}

Glaucoma is a leading cause of vision loss globally and continues to pose a challenge for clinicians and patients alike. The foundation of all current treatment options, including topical medications, lasers and surgeries, target the lowering of intraocular pressure (IOP), the only clinically validated and modifiable risk factor associated with disease progression. ${ }^{1,2}$ There has been an emergence of new surgical and medical treatment options in the last decade; ${ }^{3,4}$ however, many patients do not achieve adequate IOP lowering and many still exhibit disease progression despite maintenance of an IOP in the normal range. ${ }^{1}$ The introduction of minimally 
invasive glaucoma surgery (MIGS) procedures in particular has expanded treatment options and stimulated a shift toward a more interventional, personalized approach in earlier stages of the disease. ${ }^{3,5}$ However, despite the emergence of new and improved treatment options, the current armamentarium of glaucoma treatment options does not include a non-laser, non-pharmacological and non-surgical treatment option.

This study evaluated the use of the multi-pressure dial, or MPD (Equinox Ophthalmic, Inc., CA, USA), which is comprised of a pair of pressure-sensing goggles that individually encloses the periorbital regions of each eye with a separate connection on each side to a pressure-modulating pump. ${ }^{6-8}$ After the goggles are securely fitted, target negative pressure is programmed via software into the device and an individualized pressure value can be dialed in for each periorbital region. With the proper fit of the MPD, a localized, programmed change in atmospheric pressure (negative pressure) occurs within the established microenvironment. This leads to a decrease in pressure applied to the eye and a corresponding, instantaneous change to the pressure (IOP) inside the eye. ${ }^{8}$ The basic mechanism is driven by Pascal's law, which states that a pressure applied to an enclosed fluid is transmitted equally throughout the fluid. As the eye is essentially filled with a noncompressible fluid, pressures applied to or removed from the surface of the eye will be equally distributed throughout the eye.

The current treatment landscape in glaucoma does not offer a treatment option that effectively and safely provides IOP control at night. ${ }^{9}$ An unmet need exists for adjunctive or independent therapies that provide nocturnal IOP reduction as control of nocturnal IOP appears to be critical for mitigating disease progression in glaucoma, particularly in vulnerable populations such as normal-tension glaucoma (NTG). ${ }^{10,11}$ The safety of the MPD has been established with key safety parameters remaining unchanged during daytime wear lengths of 30 minutes (short-term) and 8 hours (long-term). ${ }^{6,7}$ However, the safety of the device worn overnight (eg, while sleeping) or tolerability of wear of the device on consecutive days has not been evaluated. This study aimed to evaluate the overnight safety and tolerability of the MPD in patients over a 7-day period.

\section{Methods}

\section{Subjects}

Ten subjects participated in this prospective, open-label, study. One eye of each enrolled subject was randomized to receive an application of $10 \mathrm{mmHg}$ negative pressure. The eye randomized to receive $10 \mathrm{mmHg}$ of negative pressure was designated as the study eye and the contralateral eye served as the intra-subject control and was denoted the control eye. Subject and demographic characteristics are summarized in Table 1. This study was performed at a single site (Sioux Falls, SD) and was approved by the Aspire IRB (Santee, California). All procedures conducted were in accordance with the Aspire IRB and the 1964 Helsinki declaration. Prior to starting the study, informed consent was obtained for each subject. This study was designated as a non-significant risk (NSR) clinical study by the IRB and did not require clinical trial registration.

Key inclusion criteria were subjects $\geq 18$ years of age with a documented diagnosis of open-angle glaucoma $(\mathrm{OAG})$ with orbital anatomy that allowed a proper and secure, air-tight seal when the goggles were placed and capable of tolerating IOP measurements with the device in place. Subjects with prior refractive surgery (eg, PRK, LASIK, SMILE), cataract surgery, cataract surgery with minimally invasive glaucoma surgery (MIGS), standalone MIGS, trabeculectomy or implantation of other glaucoma drainage devices were permitted to enter the study. Exclusion criteria included the presence or history of any eye condition/disease that could compromise the

Table I This Table Demonstrates the Subject Demographics and Baseline Characteristics of the Study Population

\begin{tabular}{|c|c|}
\hline \multicolumn{2}{|l|}{ Parameter } \\
\hline Age, years (Mean, SD) & $55.5 \pm 14.8$ \\
\hline Gender (M/F) & $8 \mathrm{M} / 2 \mathrm{~F}$ \\
\hline Ethnicity & Non-hispanic or Latino/White \\
\hline \multicolumn{2}{|l|}{ BCVA (mean $\pm S D)$} \\
\hline Study eye & $0.0 \pm 0.1$ \\
\hline Fellow eye & $0.0 \pm 0.2$ \\
\hline \multicolumn{2}{|l|}{ Glaucoma Medication use } \\
\hline 0 medications, n (\%) & $5(50 \%)$ \\
\hline I medication, $\mathrm{n}(\%)$ & $3(30 \%)$ \\
\hline 2 medications, $\mathrm{n}(\%)$ & $2(30 \%)$ \\
\hline \multicolumn{2}{|l|}{ Prior Surgery } \\
\hline None, n (\%) & $6(60 \%)$ \\
\hline SLT, n (\%) & I (10\%) \\
\hline Cataract surgery, n (\%) & I (10\%) \\
\hline Cataract + MIGS procedure, n (\%) & $2(20 \%)$ \\
\hline
\end{tabular}

Abbreviations: BCVA, best-corrected visual acuity; MIGS, minimally invasive glaucoma surgery; SLT, selective laser trabeculoplasty. 
evaluation of the study results or subject safety. Subjects with macular degeneration, retinal detachment or other fundus findings that could prevent visualization of the retina in either eye, eyelid edema or conjunctival chemosis in either eye, history of corneal transplant in either eye, or history of allergy to any of the testing materials (eg, silicone) were excluded. Women who were pregnant or lactating during the time of the study were also excluded.

\section{Study Design}

Subjects who passed screening and met inclusion/exclusion criteria were enrolled into the study and one eye of each enrolled subject was randomized to negative pressure application in one eye and zero negative pressure in the other eye. The eye randomized to negative pressure was denoted the study eye while the intra-subject contralateral eye was referred to as the control eye. Each subject was screened for proper fit of the MPD to ensure a proper seal could be achieved and maintained. All baseline testing occurred prior to wear of the goggles.

All subjects underwent a baseline ophthalmological exam. The exam included the following clinical testing: best-corrected visual acuity (BCVA), manifest refraction, OCT of the retinal nerve fiber layer (RNFL), and intraocular pressure (IOP). In addition, a comprehensive slit lamp and dilated fundus examination were performed.

For the IOP measurements, two methods were utilized for measurement: Goldmann applanation tonometry (GAT) and Reichert Model 30 pneumatonometer (Reichert Inc., Buffalo, NY; PTN). Prior to the study period, IOP measurements were obtained for both eyes with GAT and PTN. After baseline testing was completed, the study period started with the application of negative pressure with both the MPD and a modified version of the MPD known as the excursion MPD. The MPD and the excursion MPD, which includes an access port in the lens of each goggle to permit PTN measurements, are shown in Figures 1 and 2. With the excursion MPD placed and secured, measurements initially occurred with no negative pressure and then were repeated with $10 \mathrm{mmHg}$ of negative pressure applied in the study eye. This step was included as a safety and validation measure to ensure the IOP with $10 \mathrm{mmHg}$ negative pressure application over the course of the 7-day study period was not harmful to the subject. IOP measurements while negative pressure was active were obtained via the excursion test method, a method previously described in detail that permits IOP measurements while the negative pressure environment is

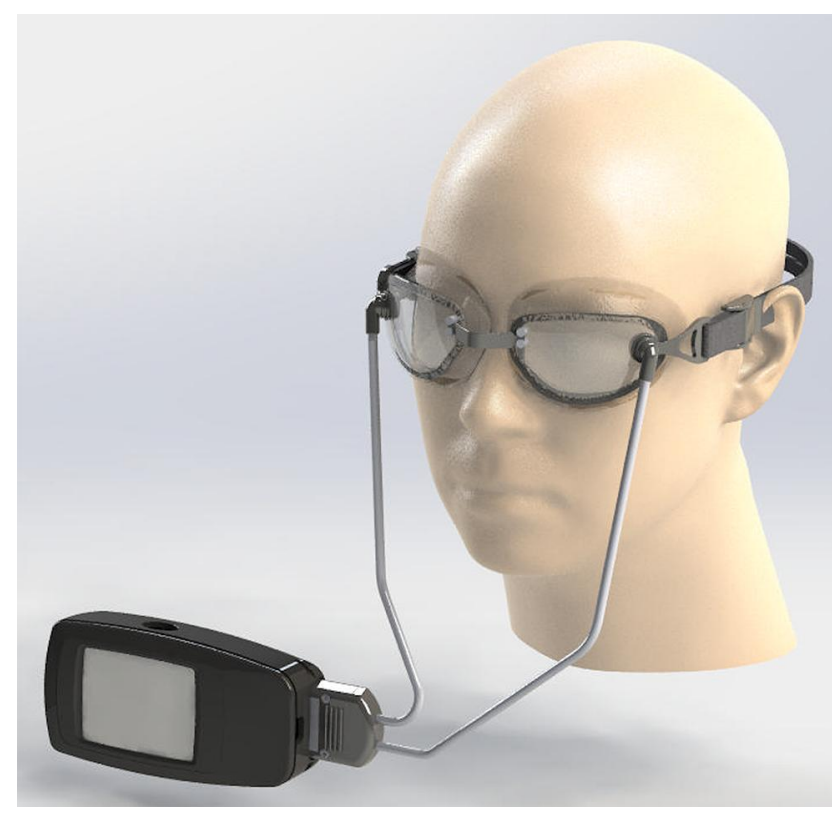

Figure I This figure demonstrates the multi-pressure dial, which includes the goggles connected to a pressure-modulating pump. This device was worn nightly by subjects for 7 consecutive nights during the study.

maintained. ${ }^{12}$ After the IOP measurements were obtained via the port in the excursion MPD, the device was removed and subjects were sent home with a standard MPD programmed for $10 \mathrm{mmHg}$ negative pressure in the study eye only. Subjects were encouraged to wear the device nightly and were educated on how to use the device and troubleshoot if necessary.

Subjects were asked to complete a daily subjective assessment, scaled 1-10 with 1 being most favorable and 10 being least favorable each morning after wear of the device that evaluated the comfort and tolerability of the device. Subjects were permitted to discontinue study participation at any time.

A summary of the visit schedule with the diagnostic testing and intraocular pressure measurements and when they were performed is included in Table 2. This study included up to a total of four study site visits. The screening visit, or visit 1, occurred in the 7 days leading up to the first day of testing, also known as visit 2. After visit 2, subjects were sent home with the MPD and instructed to start nightly wear for a 7-day period. Subjects were asked to return for a mid-term visit (between days 2-4), also known as visit 3, for a safety check that included BCVA, manifest refraction and IOP via GAT. Following the 7-day period, subjects returned for a follow-up visit (visit 4). Visit 1 (screening) and visit 2 (testing) were permitted on the same day. 


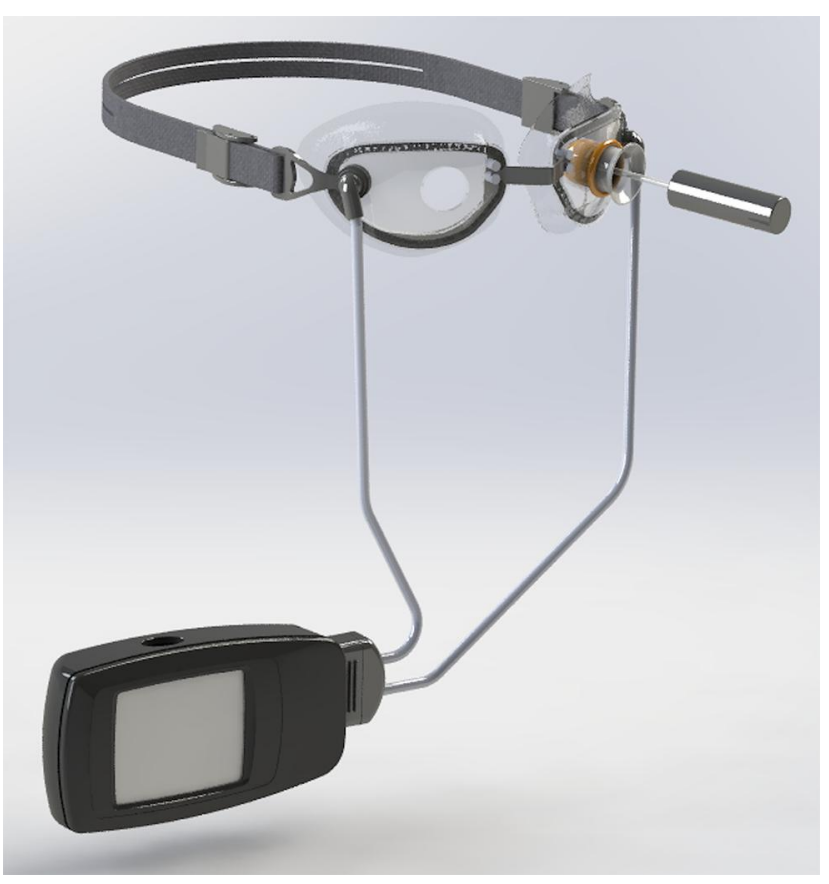

Figure 2 This figure demonstrates the excursion MPD, a modified version of the MPD which includes an access port in the goggle lenses to permit pneumatonometry measurements while negative pressure is being applied.

At visit 4, baseline testing was repeated including a dilated fundoscopic examination, OCT RNFL measurements as well as both GAT and PTN measurements of IOP. Subjects were also assessed for adverse events that may have occurred following the 7-day duration of nightly application of negative pressure.

\section{Study Materials}

The MPD, shown in Figure 1, consists of two distinct components: a pair of specialized goggles and corresponding programmable pump. The two devices are mechanically and pneumatically connected with a connector and tubing system. Collectively, the device employs vacuum (negative pressure) technology to reduce the IOP while the device is worn.

The goggles include an adjustable head strap for support as well as an adjustable nose bridge for comfort. The goggles are designed with an anthropometric fit to allow for extended wear with active negative pressure and are available in various sizes to accommodate differences in subject facial anatomy.

The MPD pump connects to the goggles via crushresistant tubing with separate lumens to allow independent negative pressure settings for each eye. The tubing system has a separate vacuum and pressure-sensing lines to allow closed-loop control of the vacuum. This design allows for real-time titratability of the negative pressure as well as pressure sensors directly in the goggle cavity to ensure the target negative pressure is reached and maintained. With a secure fit and a negative pressure value programmed into the device, a negative pressure environment is established in the microenvironment around the eye. The lowered atmospheric pressure translates to a reduction in IOP by Pascal's law and the principles of hydrostatic physics.

A modified version of the MPD, known as the excursion MPD (Figure 2), was used in this study at the study site to obtain IOP measurements while negative pressure was active.

\section{Statistical Analysis}

Paired, parametric t-tests were employed to compare the mean IOP and BCVA at baseline with subsequent visits. An $\alpha$ level of 0.05 was considered statistically significant. All statistical analyses were performed using SciPy python 3.6.7 statistical software.

\section{Results}

\section{Subject Demographics}

This study evaluated 20 eyes from 10 subjects, including 10 studies and 10 control eyes. The contralateral eye in each subject was used as the control. The mean age of the subjects was $55.5 \pm 14.8$. Eight of the 10 subjects were male. All subjects included in the study had a prior diagnosis of mild OAG. Half $(n=5)$ of the subjects were on $\geq 1$ medication prior to the study period. This demographic information is included with other preoperative characteristics in Table 1.

\section{IOP Results}

The IOP results are demonstrated in Figure 3. At baseline, IOP measurements were performed with both GAT and PTN. Baseline GAT IOP was $17.1 \pm 4.2 \mathrm{mmHg}$ in the study eye and $17.0 \pm 3.2 \mathrm{mmHg}$ in the control eye. Prior to placement of the MPD, the mean baseline IOP via PTN of the study and control eyes was $18.0 \pm 3.5 \mathrm{mmHg}$ and $18.4 \pm 4.2 \mathrm{mmHg}$, respectively. IOP measurements with the excursion MPD placed but without application of negative pressure demonstrated a mean IOP of $18.2 \pm 3.8 \mathrm{mmHg}$ in the control eye and $18.1 \pm 2.7 \mathrm{mmHg}$ in the study eye. With the excursion MPD placed and $10 \mathrm{mmHg}$ of negative pressure applied (study eye only), the mean IOP was $14.0 \pm 2.1$ in the study eye and $17.9 \pm 4.2$ in the control eye. The $23 \%$ IOP reduction 
Table 2 This Table Demonstrates the Results of the Daily Subjective Assessment That Was Completed by Subjects

\begin{tabular}{|c|c|c|c|c|c|c|c|c|}
\hline \multicolumn{9}{|l|}{ Daily Subjective Assessment } \\
\hline \multirow[t]{2}{*}{ Question } & \multicolumn{8}{|c|}{ Scale: $\mathbf{I}=$ Most Favorable, $10=$ Least Favorable } \\
\hline & Day I & Day 2 & Day 3 & Day 4 & Day 5 & Day 6 & Day 7 & Overall \\
\hline How are you tolerating the MPD? & $4.2 \pm 3.2$ & $3.1 \pm 2.4$ & $2.9 \pm 2.2$ & $2.6 \pm 1.7$ & $3.4 \pm 1.7$ & $3.0 \pm 1.6$ & $2.9 \pm 1.6$ & $3.2 \pm 0.5$ \\
\hline $\begin{array}{l}\text { How comfortable/uncomfortable } \\
\text { is the MPD? }\end{array}$ & $4.7 \pm 3.2$ & $3.4 \pm 2.5$ & $3.0 \pm 2.1$ & $3.3 \pm 1.6$ & $3.5 \pm 1.7$ & $3.1 \pm 1.4$ & $2.9 \pm 1.6$ & $3.4 \pm 0.6$ \\
\hline $\begin{array}{l}\text { How acceptable was the MPD } \\
\text { negative pressure? }\end{array}$ & $3.2 \pm 2.9$ & $2.9 \pm 2.9$ & $3.0 \pm 2.6$ & $2.9 \pm 2.5$ & $2.9 \pm 2.1$ & $2.7 \pm 2.2$ & $2.6 \pm 2.1$ & $2.9 \pm 0.2$ \\
\hline $\begin{array}{l}\text { What is the likelihood of you } \\
\text { wearing the device every night? }\end{array}$ & $3.9 \pm 2.8$ & $3.0 \pm 2.5$ & $2.9 \pm 2.2$ & $3.4 \pm 2.5$ & $3.8 \pm 2.6$ & $3.9 \pm 2.8$ & $3.4 \pm 2.6$ & $3.5 \pm 0.4$ \\
\hline $\begin{array}{l}\text { What is your attitude towards } \\
\text { the MPD as a glaucoma therapy? }\end{array}$ & $3.9 \pm 2.7$ & $3.2 \pm 2.4$ & $3.3 \pm 2.2$ & $3.2 \pm 2.3$ & $3.4 \pm 2.3$ & $3.4 \pm 2.3$ & $3.0 \pm 2.4$ & $3.3 \pm 0.3$ \\
\hline $\begin{array}{l}\text { How do you feel about } \\
\text { continuing or stopping? }\end{array}$ & $2.4 \pm 2.2$ & $1.9 \pm 1.9$ & $1.8 \pm 1.6$ & $1.9 \pm 1.7$ & $2.0 \pm 1.7$ & $2.4 \pm 2.4$ & $1.7 \pm 1.3$ & $2.0 \pm 0.3$ \\
\hline
\end{tabular}

Notes: The scale used was I = most favorable, $10=$ least favorable and the questions included in the assessment are listed on the left. The column on the right represents the overall mean value for the 7 days.

from baseline (MPD on, without negative pressure) in the study eye was statistically significant $(\mathrm{p}<0.01)$.

Following wear of the MPD for seven consecutive days overnight, mean IOP via GAT was $17.0 \pm 5.3$ and $17.2 \pm$ $4.0 \mathrm{mmHg}$ in the study and control eye, respectively. At that visit, the mean IOP via PTN with the MPD placed without negative pressure was $16.9 \pm 4.1 \mathrm{mmHg}$ in the study eye and $16.9 \pm 4.3 \mathrm{mmHg}$ in the control eye. With negative pressure active, repeat IOPs at that visit were $13.5 \pm 3.7$ and $17.4 \pm 4.4 \mathrm{mmHg}$ in the study and control eyes, respectively. The $3.4 \mathrm{mmHg}$ reduction $(\mathrm{p}<0.01)$ from baseline at this timepoint represented a $20 \%$ reduction. There were no intraocular pressure spikes $(\geq 10 \mathrm{mmHg})$ above baseline in either group at any time point.

\section{Safety Measures}

For evaluation of visual changes, best-corrected visual acuity (BCVA) values were collected at baseline, at visit 3 , which occurred midway through the 7-day study period and the follow-up visit after completion of the 7-day period. At baseline, the mean BCVA (LogMAR) of the study and control eyes of the subjects was $0.0 \pm 0.1$ and

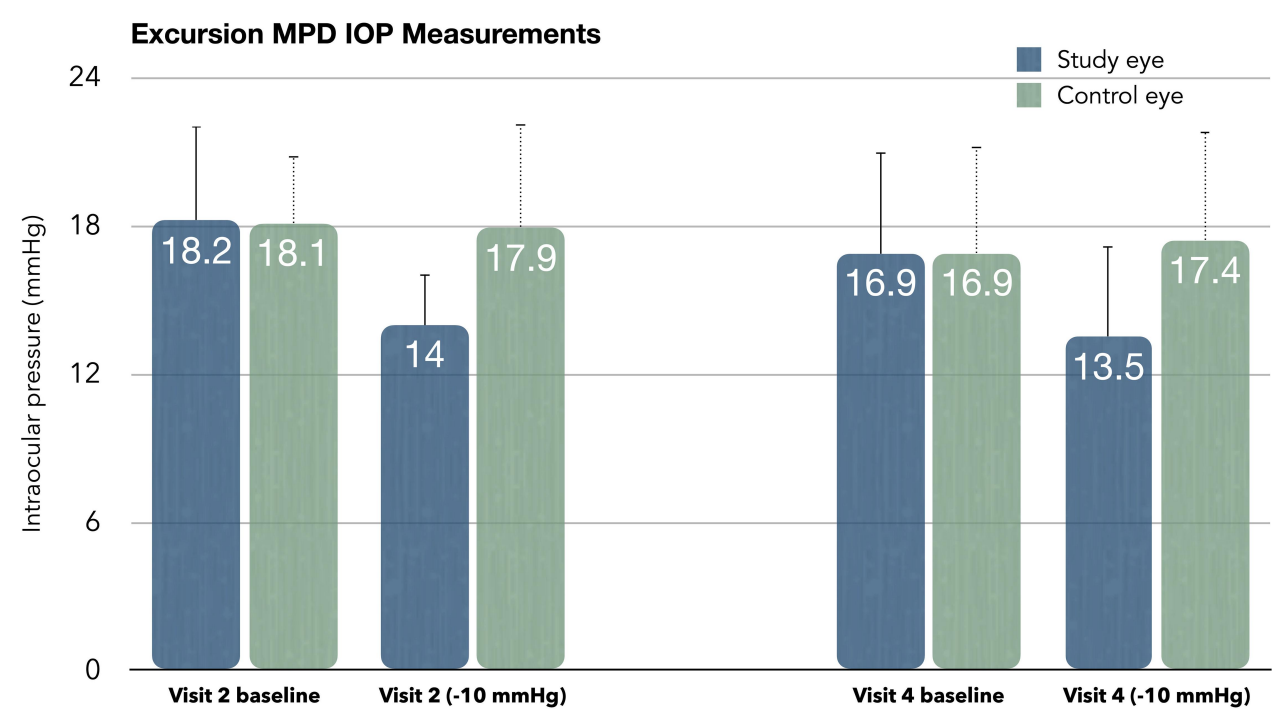

Figure 3 This figure depicts the mean IOP values obtained via pneumatonometry with the excursion MPD placed on the subject at the visit immediately prior to the 7-day wear period (visit 2) and the visit following the 7-day wear period (visit 4). Baseline values were obtained prior to application of negative pressure and negative pressure was only applied in the study eye. 
$0.0 \pm 0.1$, respectively. At visit 3 , mean BCVA was $0.0 \pm$ 0.1 in the study eye and $0.0 \pm 0.2$ in the control eye. Following wear of the device for seven consecutive days, the mean BCVA was $0.0 \pm 0.1(\mathrm{p}>0.05)$ and $0.1 \pm 0.2$ $(p>0.05)$ in the study and control groups, respectively. In summary, there were no statistically significant changes in BCVA in either the study or control eye at any time point versus baseline.

There were no observed differences from baseline in either the study or fellow eye in the slit lamp or dilated fundus examination after wear of the MPD for seven consecutive days including no evidence of optic nerve edema. In addition, there were no reported findings of conjunctival hyperemia or vessel engorgement following wear of the device.

The mean OCT RNFL global average at baseline in the study and fellow eye was $85.0 \pm 14.1$ and $87.3 \pm 20.8 \mu \mathrm{m}$, respectively. After the 7-day period, there were no significant changes from baseline with a mean RNFL global average value of $83.6 \pm 15.8 \mu \mathrm{m}$ in the study eye and $85.4 \pm 22.2 \mu \mathrm{m}$. In addition, there were no qualitative changes observed on the OCT RNFL scan at visit 3 compared to baseline.

\section{Subjective Assessment}

This study included a 6-question subjective assessment that subjects completed daily after each nightly wear of the device during the 7-day duration. The scale used for the daily assessment was $1=$ most favorable, $10=$ least favorable. The overall mean value for tolerating the MPD was $3.2 \pm 0.5$. For tolerance of the application of the negative pressure, the mean response was $2.9 \pm 0.2$. When queried about interest in continuing the treatment, the mean response was $2.0 \pm 0.3$. The complete list of questions and data from the subjective assessment are demonstrated in Table 2.

There were three adverse events reported during the 7day study period. Two subjects reported mild, periorbital edema after wear of the device that resolved without sequelae. One subject reported periorbital edema after the 6th night of consecutive wear and chose to discontinue wear of the device and thus did not complete the 7 days of consecutive wear.

\section{Discussion}

Owing to the importance of IOP control on the progression of glaucoma, IOP-lowering therapies remain the core of treatment options. ${ }^{1,13}$ Treatment decisions regarding IOP control are nearly always based on IOP measurements that occur during the day. However, IOP follows a diurnal curve and recent studies have evaluated 24-hour IOP patterns showing that, for the majority of people, peak IOP occurs nocturnally. ${ }^{14-16}$ Moreover, studies have shown that nocturnal IOP fluctuation is greater in glaucoma patients compared to healthy controls. ${ }^{17}$ The current treatment options in glaucoma do not adequately achieve nocturnal IOP control and there remains an unmet need for a safe treatment option that effectively and safely reduces IOP at night. ${ }^{18,19}$

This prospective, open-label study demonstrated the safety and tolerability of the MPD with wear of the device on consecutive nights. There were no IOP spikes above baseline in either the study or control eye noted in the follow-up visit following wear of the device for seven consecutive days, consistent with prior studies evaluating the device. ${ }^{6,7}$ Results from the subjective assessment indicate the MPD is tolerable when worn overnight while sleeping and subjects would be amenable to continuous overnight wear of the device beyond the seven consecutive days evaluated in this study. These results are compelling given the known risk of nocturnal, irregular IOP elevations and indicate the device could be worn nightly, similar to a continuous positive airway pressure (CPAP) therapy for obstructive sleep apnea; notably, the design of the MPD would not preclude concomitant wear of a CPAP. ${ }^{20,21}$

IOP measurements with active negative pressure were collected prior to the 7-day period and at the follow-up visit following the 7-day period. In the study eye, there was a statistically and clinically significant reduction in IOP at both time points with the application of $10 \mathrm{mmHg}$ of negative pressure, including a $23 \%$ reduction $(4.1 \mathrm{mmHg})$ at visit 2 and a $20 \%$ reduction $(3.4 \mathrm{mmHg})$ at visit 4 . These values indicate that when the device is worn with active negative pressure, there is an instantaneous and meaningful pressure reduction. This is particularly important because, when worn at night, the MPD may represent an option for nocturnal IOP control. Furthermore, while this study did not tailor negative pressure settings based on baseline IOP measurements, the MPD's titratability is an appealing feature of the device as some patients may be subject to larger, irregular magnitudes of IOP fluctuations and would benefit from additional IOP lowering at night. ${ }^{22}$

Limitations of the study include the small sample size, the seven-day duration, and the relative magnitude of the applied negative pressure $(10 \mathrm{mmHg})$. The final question 
of the subjective assessment (continuing versus stopping therapy) may have contributed confusion to the subject as it was posed as a binary question with a 1-10 scale answer choice. It remains unclear what consequences, if any, the MPD may have over an extended wear for weeks or months. In addition, the impact of localized, periocular negative pressure on systemic blood pressure, particularly in vulnerable populations with pre-existing disease, remains unclear and could be explored in future investigation. If the seal of the goggles is disrupted, the established negative pressure microenvironment is lost and this allows for IOP to return to baseline. If the seal is lost, the device is capable of alerting the subject with an audible/vibratory signal.

Overall, the results of this study are favorable, indicating the device can safely and comfortably be worn at night and that subjects would agree to longer, continuous wear of the prescribed device. The IOP results further confirm the IOP-lowering capability of the device, as previously observed. Future studies with long-term follow-up are necessary for investigating the impact and safety of longterm wear. Further investigation comparing the difference in efficacy and response to negative pressure using the MPD in eyes with and without a history of prior glaucoma surgery are also needed.

\section{Conclusion}

In summary, this study demonstrates that the delivery of negative pressure using the MPD is safe and tolerable for patients nightly for up to seven consecutive days. The results of the subjective assessment suggest that patients would be amenable to longer-term wear of the device beyond a 7-day period. Moreover, the significant reduction in IOP achieved through the application of negative pressure suggests the MPD may represent a future treatment option capable of nocturnal IOP control.

\section{Data Sharing Statement}

The data set collected and analyzed for this present study is available from the corresponding author per reasonable request.

\section{Acknowledgments}

This study was sponsored by Equinox Ophthalmic, Inc. (Newport Beach, CA). Article-processing charges were funded by Equinox Ophthalmic, Inc. All authors had complete access to the study data and assume full responsibility for the integrity of the data and the accuracy of the data analysis. All named authors meet the International Committee of Medical Journal Editors (ICMJE) criteria for authorship for this article, take responsibility for the integrity of the work as a whole, and have given their approval for this version to be published. We are grateful to the study participants for their participation in this study.

\section{Disclosure}

Drs. Ferguson, Radcliffe, Van Tassel, Baartman, Lindstrom are consultants for Equinox Ophthalmic, Inc. Dr. Thompson is a shareholder for Equinox Ophthalmic, Inc. Dr. Ibach is a shareholder for Equinox LLC. Dr. Berdahl is the CEO and founder of Equinox Ophthalmic, Inc and has a patent for Equinox with royalties paid. The authors report no other conflicts of interest in this work.

\section{References}

1. Heijl A, Leske MC, Bengtsson B, et al. Reduction of intraocular pressure and glaucoma progression: results from the Early Manifest Glaucoma Trial. Arch Ophthalmol. 2002;120(10):1268-1279. doi:10.1001/archopht.120.10.1268

2. Leske MC, Hyman L, Hussein M, Heijl A, Bengtsson B. Comparison of glaucomatous progression between untreated patients with normaltension glaucoma and patients with therapeutically reduced intraocular pressures. The effectiveness of intraocular pressure reduction in the treatment of normal-tension glaucoma. Am J Ophthalmol. 1999;127(5):625-626.

3. Shah M. Micro-invasive glaucoma surgery - an interventional glaucoma revolution. Eye Vis Lond Engl. 2019;6(1):29. doi:10.1186/ s40662-019-0154-1

4. Weinreb RN, Ong T, Sforzolini BS, Vittitow JL, Singh K, Kaufman PL. A randomised, controlled comparison of latanoprostene bunod and latanoprost $0.005 \%$ in the treatment of ocular hypertension and open angle glaucoma: the VOYAGER study. $\mathrm{Br} J$ Ophthalmol. 2014;99(6):bjophthalmol-2014-305908-745. doi:10.1136/bjophthalmol-2014-305908

5. Conlon R, Saheb H, Ahmed IIK. Glaucoma treatment trends: a review. Can J Ophthalmol. 2017;52(1):114-124. doi:10.1016/j. jcjo.2016.07.013

6. Thompson VM, Ferguson TJ, Ahmed IIK, et al. Short-term safety evaluation of a multi-pressure dial: a prospective, open-label, nonrandomized study. Ophthalmol Ther. 2019;82(11):887-889. doi:10.1007/s40123-019-0181-y

7. Samuelson TW, Ferguson TJ, Radcliffe NM, et al. 8 hrs safety evaluation of a multi-pressure dial in eyes with glaucoma: prospective, open-label, randomized study. Clin Ophthalmol Auckl NZ. 2019;13:1947-1953. doi:10.2147/OPTH.S217736

8. Ethier CR, Yoo P, Berdahl JP. The effects of negative periocular pressure on intraocular pressure. Exp Eye Res. 2020;191:107928. doi:10.1016/j.exer.2020.107928

9. Orzalesi N, Rossetti L, Invernizzi T, Bottoli A, Autelitano A. Effect of timolol, latanoprost, and dorzolamide on circadian IOP in glaucoma or ocular hypertension. Invest Ophthalmol Vis Sci. 2000;41 (9):2566-2573.

10. Agnifili L, Mastropasqua R, Frezzotti P, et al. Circadian intraocular pressure patterns in healthy subjects, primary open angle and normal tension glaucoma patients with a contact lens sensor. Acta Ophthalmol (Copenh). 2015;93(1):e14-21. doi:10.1111/aos.12408 
11. De Moraes CG, Jasien JV, Simon-Zoula S, Liebmann JM, Ritch R. Visual field change and 24-hour IOP-related profile with a contact lens sensor in treated glaucoma patients. Ophthalmology. 2016;123 (4):744-753. doi:10.1016/j.ophtha.2015.11.020

12. Ferguson TJ, Knier CG, Chowdhury UR, et al. Intraocular pressure measurement with pneumatonometry and a tonometer tip cover. Ophthalmol Ther. 2020;9(1):127-137. doi:10.1007/s40123-020-00235-z

13. Leske MC, Heijl A, Hussein M, Bengtsson B, Hyman L, Komaroff E. Factors for glaucoma progression and the effect of treatment: the early manifest glaucoma trial. Arch Ophthalmol. 2003;121(1):48-56. doi:10.1001/archopht.121.1.48

14. Mosaed S, Liu JHK, Weinreb RN. Correlation between office and peak nocturnal intraocular pressures in healthy subjects and glaucoma patients. Am J Ophthalmol. 2005;139(2):320-324. doi:10.1016/j. ajo.2004.09.062

15. Liu JHK, Mansouri K, Weinreb RN. Estimation of 24-hour intraocular pressure peak timing and variation using a contact lens sensor. PLoS One. 2015;10(6):e0129529. doi:10.1371/journal.pone.0129529

16. Aptel F, Musson C, Zhou T, Lesoin A, Chiquet C. 24-hour intraocular pressure rhythm in patients with untreated primary open angle glaucoma and effects of selective laser trabeculoplasty. J Glaucoma. 2017;26(3):272-277. doi:10.1097/IJG.0000000000000604
17. Tojo N, Abe S, Ishida M, Yagou T, Hayashi A. The fluctuation of intraocular pressure measured by a contact lens sensor in normaltension glaucoma patients and nonglaucoma subjects. J Glaucoma. 2017;26(3):195-200. doi:10.1097/IJG.0000000000000517

18. Liu JHK, Medeiros FA, Slight JR, Weinreb RN. Comparing diurnal and nocturnal effects of brinzolamide and timolol on intraocular pressure in patients receiving latanoprost monotherapy. Ophthalmology. 2009;116(3):449-454. doi:10.1016/j.ophtha.2008.09.054

19. Liu JHK, Medeiros FA, Slight JR, Weinreb RN. Diurnal and nocturnal effects of brimonidine monotherapy on intraocular pressure. Ophthalmology. 2010;117(11):2075-2079. doi:10.1016/j.ophtha. 2010.03.026

20. Hoban K, Peden R, Megaw R, Halpin P, Tatham AJ. 24-hour contact lens sensor monitoring of intraocular pressure-related profiles in normal-tension glaucoma and rates of disease progression. Ophthalmic Res. 2017;57(4):208-215. doi:10.1159/000455153

21. De Moraes CG, Liebmann JM, Greenfield DS, et al. Risk factors for visual field progression in the low-pressure glaucoma treatment study. Am J Ophthalmol. 2012;154(4):702-711. doi:10.1016/j. ajo.2012.04.015

22. Kim J, Caprioli J. Intraocular pressure fluctuation: is it important? $J$ Ophthalmic Vis Res. 2018;13(2):170. doi:10.4103/jovr.jovr_35_18
Clinical Ophthalmology

\section{Publish your work in this journal}

Clinical Ophthalmology is an international, peer-reviewed journal covering all subspecialties within ophthalmology. Key topics include: Optometry; Visual science; Pharmacology and drug therapy in eye diseases; Basic Sciences; Primary and Secondary eye care; Patient Safety and Quality of Care Improvements. This journal is indexed on PubMed

\section{Dovepress}

Central and CAS, and is the official journal of The Society of Clinical Ophthalmology (SCO). The manuscript management system is completely online and includes a very quick and fair peer-review system, which is all easy to use. Visit http://www.dovepress.com/ testimonials.php to read real quotes from published authors. 